\title{
Multispectral detection circuits in special applications
}

\author{
Z. Bielecki ${ }^{1}$, W. Kolosowski ${ }^{1}$, E. Sedek ${ }^{2}$, M. Wnuk ${ }^{1}$ \& J. Wojtas ${ }^{1}$ \\ ${ }^{1}$ Military University of Technology, Poland \\ ${ }^{2}$ Telecommunication Research Institute, Poland
}

\begin{abstract}
In this paper, the first stages of receivers of optical radiation are described. Special attention was paid to the selection of a detector adequate for the range of radiation being detected. Detectors of optical radiation, including UV, visible, and IR detectors are characterised. The methods of broadening of a transmission bandwidth were also discussed. Moreover, the design criteria of low noise preamplifiers used for the mentioned detectors were presented.

Keywords: photoreceiver, noise model, low noise electronics circuits.
\end{abstract}

\section{Introduction}

Receivers of optical radiation are used in many up-to-date fields of science and technology, determining the current level of technological progress. The most important fields of their applications are in industrial automation, robotics, space technology, medicine, and in military technology.

The optical radiation range covers electromagnetic radiation longer than the gamma but shorter than millimetre waves. Optical radiation from an object (signal source) is detected with a photoreceiver. It consists of optics, a detector, a preamplifier and a signal processing system.

All objects are continually emitting radiation at a rate with a wavelengths distribution that depends on the temperature of the object and its spectral emissivity. However, free space optical signal transmission is required for most of the mentioned applications.

Then radiation is attenuated by the processes of scattering and absorption. Scattering changes direction of a radiation beam; it is caused by absorption and subsequent re-radiation of energy by suspended particles. For small particles, 
compared with the radiation wavelength, the process is known as Rayleigh scattering and exhibits a $\lambda^{-4}$ dependence. However, scattering by gas molecules is negligibly small for wavelengths longer than $2 \mu \mathrm{m}$. Also, smoke and light mist particles are usually smaller than IR wavelengths. Therefore IR radiation can penetrate further through smoke and mists than visible radiation. However, rain, fog and aerosol particles are larger and consequently they scatter IR and visible radiation to a similar degree.

Total radiation received from any object is the sum of the emitted, reflected and transmitted radiation. Objects that are not blackbodies emit only a fraction of blackbody radiation, and the remaining fraction is either transmitted or reflected, in the case of opaque objects. When the scene is composed of the objects and backgrounds of similar temperatures, the reflected radiation tends to reduce the available contrast. However, reflections of hotter or colder objects significantly affect the appearance of a thermal scene. The reflected sunlight is negligible for 8-14 $\mu \mathrm{m}$ imaging, but it is important in UV, VIS and 3-5 $\mu \mathrm{m}$ band.

In general, the $8-14 \mu \mathrm{m}$ band is preferred for high performance thermal imaging because of its higher sensitivity to ambient temperature object and its better transmission through mist and smoke [1, 2]. However, the 3-5 $\mu \mathrm{m}$ band may be more appropriate for hotter objects, or when sensitivity is less important than contrast. Also, additional differences occur, e.g. a virtue of MWIR (Medium Wave Infrared) band. There, smaller diameter optics are required to obtain a certain resolution. Furthermore some detectors may operate at higher temperatures (thermoelectric cooling) than in the LWIR (Long Wave Infrared) band. Detectors operating in this region require cryogenic cooling (about $77 \mathrm{~K}$ ).

The ultraviolet detectors have been accomplished by two different devices: the photomultiplier tube (PMT) and the solid state detectors. The PMT is a vacuum tube in which radiation falls on a photocathode, causing electrons to be emitted. Photocathode materials such as SbKCs and CsTe can be used which exhibit maximum sensitivity in the range from $400 \mathrm{~nm}$ to $235 \mathrm{~nm}$, respectively. The photon in photoemissive devices must have sufficient energy to eject electron from a photocathode material. The emitted electrons are accelerated to strike another plate, the dynode, causing the emission of a number of secondary electrons. The process is repeated several times, leading to gains of a $10^{6}$ or more. The resulting current is amplified by an external circuit. PMTs are relatively high-cost, require high-voltage power supplies, and are susceptible to magnetic fields that distort electron trajectories. Photoemissive devices produce negligible dark backgrounds at room temperatures and can be constructed to be inherently solar blind.

The alternative approach has been to use semiconductor ultraviolet detectors. In most semiconductor devices, the photon causes an electron to be transition into the conduction band. Currently, the most common devices are silicon-based CCDs where the detection process requires energies of approximately an electron volt. The detectors should have excellent sensitivity in the visible and near-IR range. These devices should be sensitive to thermally induced backgrounds at room temperatures. The semiconductor detectors made of $\mathrm{GaN}$ or other highband-gap materials, such as $\mathrm{SiC}, \mathrm{AlN}$, diamond, have the activation energy 
higher than $3.2 \mathrm{eV}$, making these devices inherently solar blind and UV sensitive without thermally induced background. While there is an increased interest in UV monitoring, the detection methods have not in general kept pace with the demand. However, looking for next generation semiconductor materials, have now led to the development of new kinds of UV detector. In particular, UV detectors based on a new material, Gallium Nitride, show very competitive properties, such as high responsivity, low dark current, very low input power requirements and good rejection of visible light. The latter is particularly important, since optical filters used to reject visible light cause complications in the design of optical systems and make it more difficult to ensure reproducible measurements. The visible rejections are 750:1 and 0.85:1 for $\mathrm{GaN}$ and $\mathrm{Si}$ detectors respectively (visible rejection $325 \mathrm{~nm}: 400 \mathrm{~nm}$ ).

Another very important benefit of $\mathrm{GaN}$ based detectors is that it is possible to tailor this wide band gap semiconductor to fit a popular UV response range. When GaN and AlN form an alloy AlGaAn, the band gap can change from 3,4 to $6,2 \mathrm{eV}$, which corresponds to the change of the optical cut-off from $365 \mathrm{~nm} 200$ $\mathrm{nm}$. Within this range a particular cut-off can be achieved by controlling the composition of the alloy.

The band gap also determines the maximum operation temperature. At high temperatures the band gap starts to collapse so that the material can no longer act as a semiconductor. The nitride based semiconductors have a band gap which is 3 to 5 times longer and in consequence, are more stable at high temperatures. Current detectors can operate well at up to $85^{\circ} \mathrm{C}$. Moreover, if material quality improves the detectors will operate at even higher temperatures. The natural rejection of visible light, high temperatures operation, low operating voltage and flexibility of the optical cut-off will allow this material to be used as a next generation tool in the field UV detection.

Typical applications UV detectors: UV curing and drying, combustion monitoring, arc detection, phototherapy, sterilization control, spectroscopy, biological agent detection, solar irradiance measurement, industrial process monitoring, missile and artillery fire detection, solar irradiance measurement, climatological and biological studies.

\section{Detection theory}

The common problem of any type of photon detector is how to terminate the photodetector with a suitable load resistor, and to trade off the performance between bandwidth and signal-to-noise ratio. This is necessary for a wide family of detectors, including phototubes, photoconductors, photodiodes, etc., all of which are described by a current generator $I_{p h}$ with a stray capacitance $C$ across it. Let us consider the equivalent circuit of a photodetector ending on a load resistor $R_{L}$, as shown in Fig. 1. This is the basic circuit for detection. The output signal in voltage $V=I R$ or in current $I$. Two noise contributions are added to the signal. One is the Johnson (or thermal) noise of the resistance $R_{L}$, and the second is the quantum noise. 


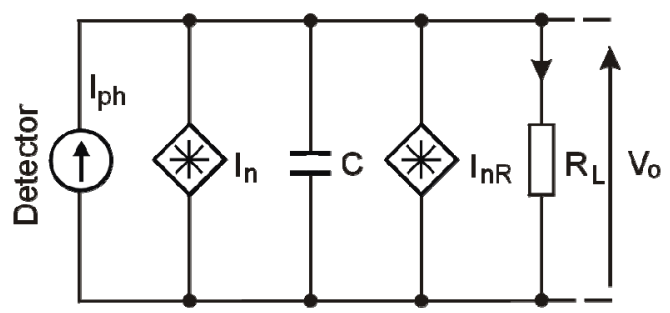

Figure 1: A general noise equivalent circuit for a photodetector.

The total current $I=I_{p h}+I_{d}$ is the sum of the signal current $\left(I_{p h}\right)$ and the dark current $\left(I_{d}\right)$. With this current is associated the quantum (or shot) noise arising from the discrete nature of electrons and photoelectrons. Its quadratic mean value is given by [3]

$$
I_{n}^{2}=2 q\left(I_{p h}+I_{d}\right) \Delta f,
$$

where $q$ is the electron charge and $\Delta f$ is the observation bandwidth.

A general noise equivalent circuit for a photodetector is shown in Fig. 1. The above two fluctuations are added to the useful signal and the corresponding noise generators are placed across the device terminals. Since the two noises are statistically independent, it is necessary to combine their quadratic mean values to give the total fluctuation as

$$
I_{n}^{2}=2 q\left(I_{p h}+I_{d}\right)+4 k T \Delta f / R_{L}
$$

The bandwidth and noise optimisation impose opposite requirements on the value of $R_{L}$. To maximise $\Delta f$ one should use the smallest possible $R_{L}$, whilst to minimise $I_{n}^{2}$ the largest possible $R_{L}$ is required. A photodetector can have a good sensitivity using very high load resistances, but then only modest bandwidths ( $\approx \mathrm{kHz}$ or less), or it can be made fast by using low load resistances (e.g. $R_{L}=50 \Omega$ ), but at the expense of sensitivity.

Using Eq. (2), we can evaluate the relative weight of the two terms in total noise current. In general, the best possible sensitivity performance is achieved when the shot noise is dominant compared to the Johnson noise, i.e.

$$
2 q\left(I_{p h}+I_{d}\right) \Delta f \geq 4 k T \Delta f / R_{L},
$$

which implies the condition

$$
R_{L \min } \geq \frac{2 k T / q}{I_{p h}+I_{d}} .
$$

At low signal levels, very high values of resistance are required; for example, for $I_{d}=5 \mathrm{pA}$, not an unusually low dark current, then $R_{L \min }=10 \mathrm{G} \Omega$. However, if we are using a resistor termination value $R_{L}<R_{\text {Lmin }}$ then, the total noise can be written as

$$
I_{n}^{2}=2 q\left(I_{p h}+I_{d}\right) \Delta f\left(1+R_{L \min } / R_{L}\right) .
$$

This means that the noise performance is degraded by factor $R_{L \min } / R_{L}$, compared to the intrinsic limit allowed by the dark current level. Thus, using $R_{L}$ 
$<R_{\text {Lmin }}$ means that the shot-noise performance is reached at a level of current not less than

$$
I_{p h}+I_{d} \geq 2 k T / q R_{L} .
$$

From this equation, we see that, for a fast photodiode with a $50 \Omega$ load at $T=300 \mathrm{~K}$, this current has very large value of $1 \mathrm{~mA}$.

The above considerations are valid for photodetectors without any internal gain, $G$. Let extend the calculation of the signal-to-noise ratio $(S / N)$ to photodetectors having internal gain. In this case, the shot noise can be expressed in the form

$$
I_{n}^{2}=2 q\left(I_{p h}+I_{d}\right) \Delta f G^{2} F,
$$

where $F$ is the excess noise factor to account for the extra noise introduced by the amplification process. Of course, in a non-amplified detector, $F=1$ and $G=1$. The total noise is a sum of shot noise and thermal noise, then we obtain a $S / N$ ratio

$$
\frac{S}{N}=\frac{I_{p h}}{\left[2 q\left(I_{p h}+I_{d}\right) \Delta f F+\left(4 k T \Delta f / R_{L} G^{2}\right)\right]^{1 / 2}} .
$$

\section{Response time of the first stage of a photoreceiver}

A response time of the first stage of photoreceiver can be shortened in many ways. Here, the possibility of such shortening in photoreceivers with $p-i-n$ photodiodes is described.

High-frequency properties of $p-i-n$ photodiodes depend on lifetime of minority carriers. In photodiodes with the Schottky barrier, lifetime of carriers is negligible $\left(\approx 10^{-14} \mathrm{~s}\right)$ when compared with minority carriers lifetime in $p-i-n$ photodiodes [4].

Response time of $p-i-n$ photodiode depends on:

- time of carriers drift through the depletion region,

- time of carriers diffusion to depletion region,

- $R C$ time constant of a load circuit of a detector.

Influences of the time $\left(\tau_{d}\right)$ of carriers diffusion to depletion region can be neglected assuming that majority of carriers are generated in a depletion region. A drift time of minority carriers through the depletion region depends on its width and carriers velocity (voltage of reverse bias). The drift time can be reduced by narrowing the depletion region. However, it causes increase in junction capacity.

A detector capacity depends on the photodiode area $(A)$, its resistivity $(\rho)$, and a voltage of reverse bias.

$$
C_{d} \propto \frac{A}{\left(V_{b}+0.5\right)^{1 / 2} \rho} .
$$

Decrease in $R C$ time constant of the first stage of a photoreceiver, the same broadening of a transmission band can be obtained by using the lower capacity photodiode and increase in a value of reverse bias voltage. However, the higher 
bias voltage can cause increase in dark current of a photodiode, i.e., the higher noise.

Next factor affecting the photodiode response time $\left(t_{r}\right)$ depends mainly on its capacity and input resistance of a preamplifier. A time constant of this circuit is

$$
\tau_{R C}=\frac{\left(R_{L}+R_{S}\right) R_{s h}}{R_{L}+R_{s}+R_{s h}} C=R_{e q} C,
$$

where $R_{s}$ is the series resistance of a photodiode, $C$ is the sum of photodiode capacity and input capacity of a preamplifier, $R_{e q}$ is the resultant resistance which is a parallel connection of the resistance $R_{s h}$ of photodiode and the load resistance $R_{L}$.

A response time and transmission bandwidth can be shaped by selection of a capacity of the first stage of a photoreceiver. Decrease in a width of a depletion region causes reduction of photodiode sensitivity, the same possibility of detection of optical signals of low amplitudes. Thus, the compromise between a response time of a photoreceiver and its sensitivity is necessary.

Minimize $\mathrm{C}_{\mathrm{d}}+\mathrm{C}_{\mathrm{i}}$ :

- small photodiode area,

- Reverse bias,

- low $\mathrm{C}_{\mathrm{i}}$ opamp

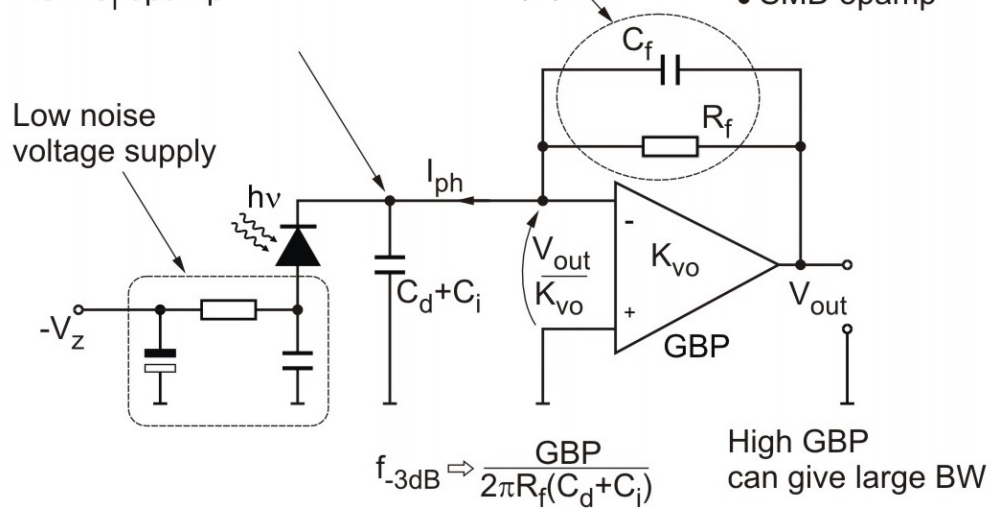

Minimize $\mathrm{C}_{\mathrm{f}}$ :

- split resistors $R_{f}$

- compact layout

- SMD opamp

Figure 2: Factors affecting the response time of the first stage of a photoreceiver.

The above considerations show, that the detector response time is

$$
t_{T}=\left(t_{r}^{2}+t_{t}^{2}+t_{d}^{2}\right)^{1 / 2},
$$

and $3 \mathrm{~dB}$ limit frequency is given as

$$
f_{-3 d B}=\left(2 \pi R_{e q} C\right)^{-1} \text {. }
$$

Usually, a designer can obtain the broadening of a transmission bandwidth of a photoreceiver when a detector of low capacity and preamplifier of low input resistance are used. 
Figure 2 presents the factors influencing response time of the first stage of a photoreceiver. Of course broadening of a transmission bandwidth of the first stage of a photoreceiver will cause noise increase, i.e., decrease in its sensitivity.

\section{Selection of the first stage of a photoreceiver}

As an active element in a preamplifier, the bipolar or FET transistor or integrated system, with an input at a bipolar transistor, FET transistor or MOSFET transistor, can be used. The main criteria of selection are both the value of detector resistance and the range of transmitted frequencies [5-7].

Because low intensity signals reach a photoreceiver, a very important task is to optimize the noise of a photodetector-preamplifier, i.e., to obtain maximum of $\mathrm{S} / \mathrm{N}$ ratio. The first stages of complex electronic devices significantly affect the level of a total noise of a system, so preamplifiers have to fulfil very special requirements. Optimum parameters of a preamplifier can be determined by the basis of analysis of particular sources of the noise in a total equivalent input noise of a photodetector-preamplifier system and on the basis of calculation of an equivalent input noise. A level of an equivalent noise at the input of a photodetector-preamplifier is univocally determined by the detector noise $\mathrm{V}_{\mathrm{nd}}$, background noise, and equivalent noise sources $V_{n}$ and $I_{n}$. For non correlated components of noise, the total equivalent noise at the input of a photodetectorpreamplifier is defined as

$$
V_{n i}^{2}=V_{n d}^{2}+V_{n b}^{2}+V_{n}^{2}+I_{n}^{2} R_{d}^{2},
$$

where $R_{d}$ is the detector resistance.

It can be generally stated that if high level of current noise is in a transistor of the first stage of a preamplifier, this transistor cannot operate with highresistance detector. High level of the current noise of input transistor causes high equivalent input noise at high resistance of a detector. On the contrary, lowresistance detector can well operate with low voltage noise preamplifier.

In $[1,3]$, detailed analyses have been performed on selection of preamplifiers for various detectors of optical radiation. For optical radiation detectors, the voltage preamplifiers, transimpedance preamplifiers, and charge preamplifiers are used. High signal-to-noise ratio in a voltage preamplifier causes narrowing of a transmission bandwidth of a system. Due to application of a transimpedance preamplifier for the determined detector resistance, the higher bandwidth can be obtained. Charge preamplifiers form a separate group.

Figure 3 presents a simplified scheme of the first stage of a photoreceiver with transimpedance preamplifier and noise sources. Such a system is commonly used for UV, VIS and IR detectors, both photoconductive detectors and photodiodes.

Improvement in this $\mathrm{S} / \mathrm{N}$ ratio can be obtained by:

- decrease in influence of background radiation (narrowed field of view of a detector due to applied cooled diaphragms and optical filters),

- decrease in generation-recombination noise produced from thermally induced carriers in semiconductor (lower temperature of detector operation),

- decrease in thermal noise of a detector (cooling), 


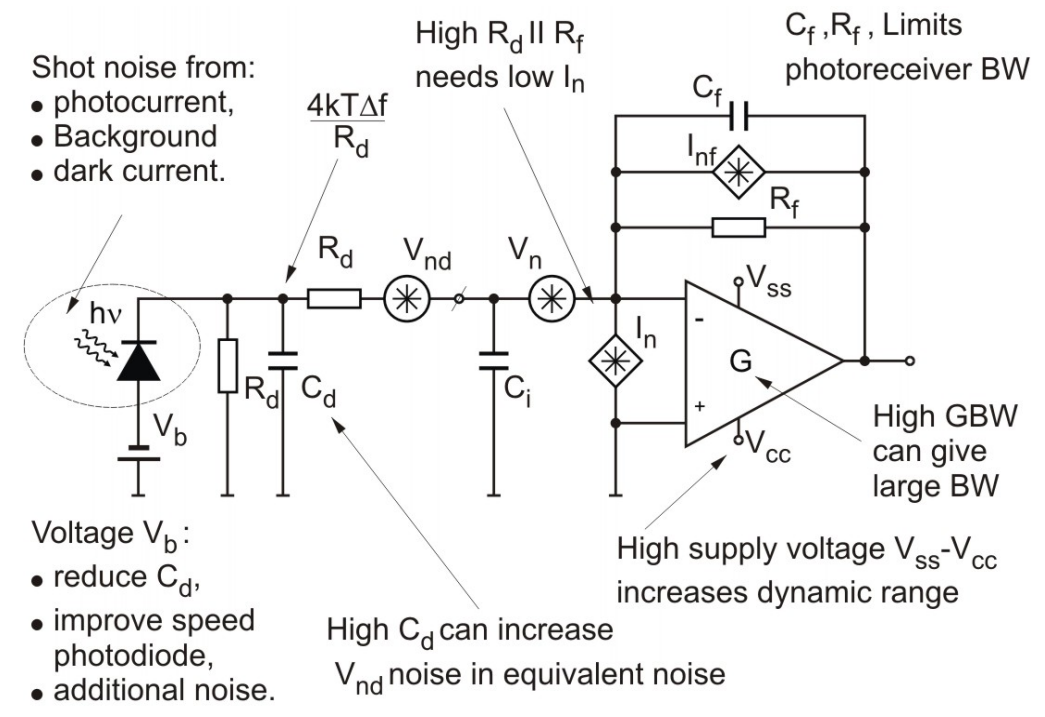

Figure 3: Simplified equivalent scheme of the first stage of a photoreceiver with noise sources.

- decrease in thermal noise of load resistance (using high $R_{L}$ values and in some applications additional decrease in detector operation temperature),

- use of the optimised ultra low-noise preamplifier [8-11].

\section{Experimental results}

For amplification of the signals from UV detectors, the most frequently voltage and transimpedance amplifiers are used. The basic idea of increase in input impedance of preamplifier is reduction of thermal noises. However, the high resistances $R_{L}$ cause narrowing of a band of the input stage of a photoreceiver. A preamplifier of high input impedance is significant load resistance for a detector, so it does not ensure wide range of signal changes. The problem of serious changes of a signal has been solved in transimpedance preamplifiers.

For amplification of the signals from UV detectors, we used transimpedance preamplifier. In the preamplifier the integrated circuit of AD 548, AD 549, AD 795, and OPA 129 type were used. The noise current was obtained $1.8 \mathrm{fA} / \mathrm{Hz}^{1 / 2}$, $0.5 \mathrm{fA} / \mathrm{Hz}^{1 / 2}, 0.6 \mathrm{fA} / \mathrm{Hz}^{1 / 2}, 0.1 \mathrm{fA} / \mathrm{Hz}^{1 / 2}$ respectively, for $\mathrm{f}=1 \mathrm{kHz}$.

In the case of IR detectors (e.g. HgCdTe), we used transimpedance preamplifiers too, but we optimized it on voltage noise. Integrated circuits LT 1028 and AD 797 type were used at the input stage of a preamplifier. These preamplifiers can be applied both for photodiodes and photoresistors.

When a photoresistor is connected, the biasing resistor $R_{L}$ is required. For this system the noise voltage was below $1 \mathrm{nV} / \mathrm{Hz}^{1 / 2}$, for $\mathrm{f}=1 \mathrm{kHz}$. 
Many problems have to be overcome during construction of low-noise preamplifier for low-resistance detectors (e.g., $\mathrm{HgCdTe}$ of resistance below $100 \Omega$ ).

It is due to the fact that the detectors of resistance of the order of $20 \Omega$ produce noise voltage lower than $0.6 \mathrm{nV} / \mathrm{Hz}^{1 / 2}$, i.e., below the noise voltage generated in the best (available) amplifying elements.

So, the question arises; it is possible to build a preamplifier of noise voltage below a detector noise? It appears that it can be achieved when several identical preamplifiers are connected in parallel and next the output signals are added.

Such a system of signal processing ensures reduction of final input signal according to the relationship

$$
V_{n \text { total }}=V_{n} \cdot(n)^{-1 / 2},
$$

where $V_{n}$ is the noise voltage of a single preamplifier and $n$ is the number of amplifying stages. For preamplifier with AD 797 type, the noise voltage value of $0.3 \mathrm{nV} / \mathrm{Hz}^{1 / 2}$, for $\mathrm{n}=9$ and $\mathrm{f}=10 \mathrm{kHz}$ was obtained.

\section{Example of low noise photoreceivers in military applications}

In recent years an increase of terrorism threats has occurred. It is supported by a large number of infrared guided missiles; limitation of possibilities of the airport protection, access to the airplane schedules and airplanes are an easy target (especially passenger planes). To prevent such occurrences the following security mechanisms are used: decreasing target's signature, using camouflage smoke, using pyrotechnics sources, radiation generators, multi-spectral detection systems as well as blinding systems $[13,14]$. Some of these security mechanisms have limited range of use e.g. flare shouldn't be use in the urbanized area. Flares do not meet the functions in close to attacking missiles and also do not protect airplane against the armour-piercing missiles attack.

The multi-spectral systems are free from these drawbacks e.g. active radio systems, passive infrared systems, passive ultraviolet systems. Progress in the aviation technique caused minimal altitude limit reduction up to $20-50 \mathrm{~m}$, similar causes can occurs using the rocket technique. These objects are hard to detect because of physics phenomena of the microwaves propagation in the ground zone. Based on radiolocation equation, maximal detection distance depends on direction toward the target and is written as

$$
r_{\max }(\Theta, \varphi)=r_{m o} F(\Theta, \varphi) .
$$

This formula describes the surface equation with following parameters: $r, \Theta, \varphi$, with the coordinates beginning from the radiolocation station as a reference point. This surface divides the area around radiolocation station into the two areas: area $I$, where the target is detectable and area $I I$, where target is invisible (Fig. 4a). 
Taking into consideration the ground impact it is necessary to add in radiolocation station's range in the free space equation interferometric multiplier. The radiolocation station's range is calculated using the following formula

$$
r_{\max }(\Theta)=r_{m o} F(\Theta) \Phi(\Theta),
$$

where $F(\Theta)$ - characteristics multiplier, $\Phi(\Theta)$ - interferometric multiplier.

In Fig. $4 \mathrm{~b}$ radiolocation station visibility is shown.

a)

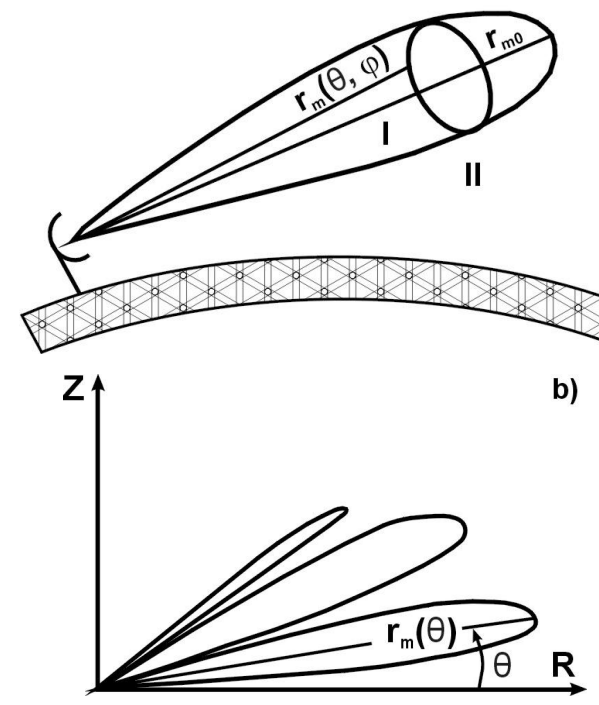

Figure 4: Radiolocation station visibility zone in the free space (a) and radiolocation station visibility (b)

Figure 4 appears a dead area. To detect the target in this area the optoelectronics systems are used. The systems operate in UV and IR spectrum region. They can be installed on the shared mast together with radiolocation antenna. The range of this system is determined using following formula

$$
d_{k m}=3,56\left(h_{a(m)}+h_{c(m)}\right),
$$

where: $h_{a}$ - hang height of the optoelectronics device in meters, $h_{c}$ - target height in meters.

In the Institute of Optoelectronics MUT original devices were elaborated. One of them is laboratory model of passive locator of flying objects.

This device includes thermodetection modules with PV $\mathrm{HgCdTe}$ detector, polish Vigo System Ltd., optimized for the spectral range of 3-4.2 $\mu \mathrm{m}$.

The passive locator can detect thermal objects from several kilometres (on the right of Fig. 5). On the left of Fig. 5 we can see these same thermal object, but in UV spectral range. In such detection systems we used photoreceiver with AlGaN detectors. 


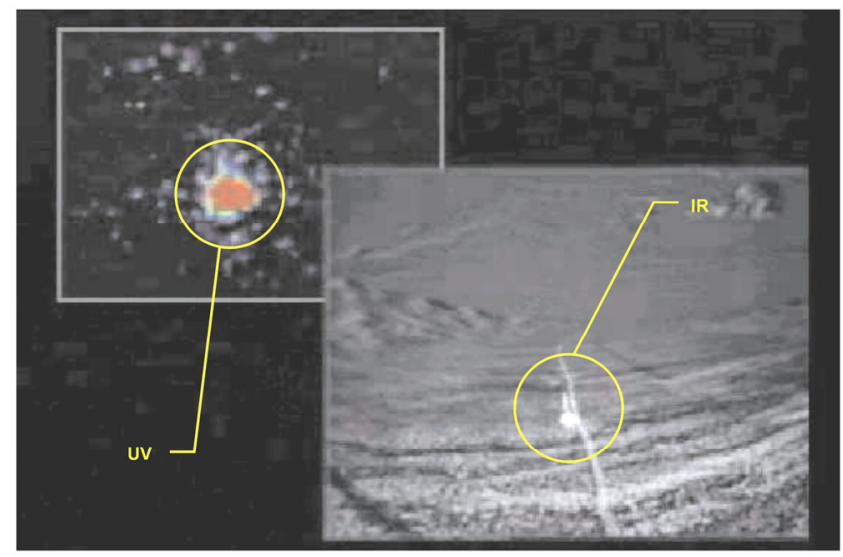

Figure 5: View of the thermal object in UV and IR wave ranges [12].

\section{Conclusions}

Increase in a signal-to-noise ratio in the first stages of photoreceivers can be obtained as a result of decrease in the noise originating from background, minimisation of a detector's noise, selection of an optimal point of detector operation, and application of low-noise preamplifier matched to detector.

Decrease in background noise can be achieved by reduction of an angle of view of a detector, application of a selective filter matched to a spectrum of a signal noise, and application of cooled choppers for long wavelength detectors.

Reduction of detector's noise is mainly due to lower operation temperature, narrower noise bandwidth of a system, and selected optimal working point.

The noise of a preamplifier in the total equivalent noise of the first stage of a photoreceiver is the lowest one in the conditions of noise matching. A value of detector resistance is a main criterion for selection of low-noise preamplifier. For low-resistance detectors, preamplifiers optimised with respect to noise voltage should be used but for high-resistance detectors with respect to noise current. Further increase in signal-to-noise ratio at the receiver output is possible due to application of adequate techniques of signal modulation and demodulation. The results of the above activities were used for experimental investigation, i.e., several optical detection devices were designed, performed and tested.

The results presented in this work do not comprise all the problems related to maximisation of $\mathrm{S} / \mathrm{N}$ ratio in optical receivers.

\section{References}

[1] Rogalski, Z. Bielecki. Chapter entitled "Detection of optical radiation", in Handbook of optoelectronics. Taylor \& Francis, New York, London pp. 73117 (2006). 
[2] Z. Bielecki, J. Mikolajczyk. Chapter entitled. "Passive infrared detection" in Photonics Handbook, Laurin Publishing, USA H123-H125, (2005).

[3] A. Rogalski, Z. Bielecki. Detection of optical radiation. Bulletin of Polish Academy of Science, vol. 52, no. 1, pp. 43-66 (2004).

[4] Z. Bielecki: "Photoreceiver with avalanche C-30645 photodiode" IEE Proceedings Optoelectronics, Vol. 147, pp. 234-236, 2000.

[5] C.D. Mothenbacher, J.A. Connelly: Low-noise electronic system design. New York, Willey, 1995.

[6] Z. Bielecki: "Maximisation of signal to noise ratio in infrared radiation receivers," Opto-electron. Rev. 10, 209-216 (2002).

[7] Z. Bielecki. Some problems of optimization of signal-to-noise ratio in infrared radiation receivers. Proc. SPIE, Vol. 5125, pp. 238-245 (2002).

[8] Z. Bielecki, M. Brudnowski. Method of popcorn-noise reduction. OptoElectron. Rev., Vol. 11, no. 1, pp. 45-50 (2003).

[9] Z. Bielecki. Readout electronics for optical detectors. Opto-Electron. Rev., Vol. 12, no. 1, pp.129-137 (2004).

[10] Z. Bielecki, W. Kolosowski, R. Dufrene, E. Sedek, J. Wojtas. Photoreceiver for BLU/UV detection. Proc. of SPIE, Vol. 5472, pp. 383-390, (2004),

[11] R. Cwirko, Z. Bielecki, J. Cwirko, L. Dobrzanski. Low-frequency noises as a tool for UV detectors characterisation. Opto-Electron. Rev., vol. 14, no 2. pp.155-160 (2006).

[12] Z. Bielecki, K. Kopczynski, M. Kwasny, Z. Mierczyk. In polish. Monitoring zagrozen bezpieczenstwa. III Międzynarodowa Konferencja Naukowa Zarzadzanie kryzysowe" Szczecin, Materiały konferencyjne, s. 310-320 (2005).

[13] Z. Bielecki, W. Kołosowski, M. Muszkowski, E. Sędek. Chapter entitled. "Phase shifters or optoelectronic delay lines application to sequential analysis of space adaptive phased arrays antennas", in Computational Methods and Experimental Measurements, WIT Press, Southampton, Boston, UK, pp.241-249 (2005).

[14] E. Sędek, Z. Bielecki, M. Muszkowski, W. Kołosowski, G. Różański, M. Wnuk. „Optoelectronic system for phase array antenna beam steering” in Computational Methods and Experimental Measurements, WIT Press, Southampton, Boston, UK, pp. 801-808 (2007). 